\title{
Qualitätssicherung für wissenschaftliche Veröffentlichungen
}

\section{Für eine differenzierte und fachgerechte Betrachtung disziplinärer Publikationskulturen}

\author{
Erarbeitet von Sven Stollfuß ${ }^{1}$ (D), Christoph Schindler ${ }^{3}$ D, Klaus Rummler ${ }^{2}$ (D), \\ Petra Missomelius ${ }^{1}$ (D) und Theo Hug² (D) \\ ${ }^{1}$ für die Gesellschaft für Medienwissenschaft (GFM) \\ ${ }^{2}$ für die Sektion Medienpädagogik der Deutschen Gesellschaft für Erziehungswissen- \\ schaft (DGfE) \\ ${ }^{3}$ DIPF Leibniz-Institut für Bildungsforschung und Bildungsinformation
}

\section{Zentrale Forderungen}

1. Die Bewertung wissenschaftlicher Leistungen (etwa bei Drittmittelanträgen, Berufungen oder Evaluationen) muss auf der Basis der Inhalte selbst und nicht entlang intransparenter und wenig aussagekräftiger (Publikations-)Kennzahlen erfolgen.

2. Wir appellieren an alle Beteiligten im Wissenschaftsbetrieb, in der Hochschulund Forschungsförderpolitik, die Offenheit fachlich diverser Veröffentlichungstraditionen und -kulturen anzuerkennen und gleichwertig zu berücksichtigen.

3. Für eine offene Diskussion der Bedeutung von heterogenen Publikationskulturen im Wissenschaftsbetrieb fordern wir die Einrichtung interdisziplinärer Reformkommissionen mit Vertreterinnen und Vertretern geistes-, kultur- und sozialwissenschaftlicher Fachgesellschaften, der Hochschulleitungen, der zuständigen Ministerien und des Wissenschaftsrats.

Die Gesellschaft für Medienwissenschaft und die unterstützenden Fachgesellschaften beobachten mit Sorge die sich seit einigen Jahren abzeichnenden Transformationsdynamiken in der Diskussion um die Beurteilung wissenschaftlicher Publikationen. Grundsätzlich erfreulich ist, dass das Publizieren in den Geistes- und Kulturwissenschaften sowie in Teilen der Bildungs- und Sozialwissenschaften an eine international 
vernetzte Publikationskultur anschliesst. Zu bedenken ist dabei allerdings, dass verstärkt Massstäbe einer quantitativen Vergleichbarkeit wie Impact Factor ${ }^{1}$ oder $h$-Index ${ }^{2}$ fast alle fachlichen Disziplinen in zunehmender Weise durchdringen. ${ }^{3}$ Diese Mechanismen haben sich an vielen Hochschulen zu dominanten Qualitätskriterien und relevanten Instrumenten der Steuerung von Wissenschaft entwickelt, auf deren Grundlage Finanzierungs-, Förder- und Berufungsentscheidungen getroffen werden. Hierdurch entsteht unweigerlich eine kollektive Abhängigkeit von intransparenten Bewertungsinstrumenten gewinnorientierter Unternehmen, die die Wissenschaftsfreiheit langfristig nachteilig beeinflusst und akademische Diskurskulturen normiert. In der Veröffentlichungspraxis ist es grundsätzlich sinnvoll und zielführend, durch begleitende Begutachtungen zu einer Qualitätssicherung von Wissenschaft beizutragen. Im Zusammenhang mit den intransparenten Messkriterien grosser Verlage und kostenpflichtiger Datenbanken werden allerdings auch Steuerungseffekte erzeugt, die Dynamiken des Mainstreamings, der Normierung und der Ungleichverteilung von Ressourcen befördern. Die damit verbundene Einschränkung fachlicher Autonomie zeigt sich in besonderer Weise in der Reduktion des Spektrums relevanter Qualitätskriterien auf exkludierende und intransparente Parameter und die damit korrespondierenden Mechanismen. Traditionsreiche Publikationen und Zitationen in Sammelbänden namhafter Verlage sowie in nicht-indizierten Fachzeitschriften figurieren in diesen Systemen so wenig wie ‘graue` oder nicht-digitalisierte Literatur.

Für die Berufung von Professorinnen und Professoren, die Evaluation von Juniorprofessorinnen und -professoren sowie für Leistungsvereinbarungen (z. B. Tenure Track) schreiben sich diese Beurteilungsmassstäbe zunehmend in die Bewertung von Publikations- und Forschungsaktivitäten ein. Teils vor dem Hintergrund von Vorgaben durch Wissenschafts- und Bildungsministerien und universitätsinterner Regularien, teils durch die Festlegungen in den jeweiligen Begutachtungskommissionen avanciert die Bewertung anhand intransparenter Metriken zu entscheidenden Faktoren vermeintlicher Objektivität. Zum Ersten liegt hier ein häufig übergangener intransparenter Interessenkonflikt vor, der darin besteht, dass Produktions-, Distributions- und Bewertungsinfrastrukturen in den gleichen Händen liegen: So betreibt z. B. die RelX Group neben Scopus diverse Datenstrukturen und Angebote zur Bewertung und Verbreitung von Publikationen und gleichzeitig unter dem Markennamen

1 D. h. der Zahlenwert, der sich auf den errechneten wissenschaftlichen Einfluss eines Fachjournals aufgrund dessen durchschnittlicher Zitierhäufigkeit pro Jahr bezieht.

2 D. h. die bibliometrisch errechnete Zitierhäufigkeit einzelner Autorinnen und Autoren, abhängig von der Anzahl von Artikeln in spezifischen Fachdatenbanken, die wiederum Teil von zumeist kostenpflichtigen Recherchediensten wie dem Web of Science oder Scopus sind.

3 Zur Kritik am Journal Impact Factor siehe auch die San Francisco Declaration on Research Assessment (https://sfdora.org), die schon 2012 die intransparenten, feldspezifisch nicht vergleichbaren und manipulationsanfälligen Metriken wie dem Impact Factor als ungeeignet zur Bewertung von Ergebnissen der wissenschaftlichen Forschung eingestuft hat (vgl. dazu auch die Hong Kong Principles, https://wcrif.org/ guidance/hong-kong-principles). 
Elsevier den entsprechenden Verlag. Zum Zweiten wird mit kommerziellen Zitationsdatenbanken (z. B. Social Sciences Citation Index oder Arts and Humanities Citation Index) eine strukturelle Engführung «begutachtungs- oder evaluationsrelevanter» wissenschaftlicher Leistungen hergestellt, deren Normierungseffekte im deutschsprachigen Raum bislang kaum reflektiert wurden.

Im Zuge der daraus folgenden künstlichen Verknappung der Diversität von Publikationsformen über Prozesse der Standardisierung werden in der Praxis nicht-englischsprachige Veröffentlichungen sowie tradierte Publikationsformen wie Monographien, Sammelwerke oder auch Festschriften in ihrer Bedeutung unterbewertet und zunehmend ausgegrenzt. Damit verbunden ist ferner ein zunehmendes Ausspülen fachbegrifflicher Genauigkeiten, das in nicht-englischsprachigen theoretisch und philosophisch orientierten Forschungsbereichen zum Nachteil gereicht, wenn der wissenschaftliche Diskurs einerseits sprachlich und andererseits auch inhaltlich zu vereinheitlichen droht.

Die zunehmende Fokussierung auf profitorientierte und kommerziell indexierte Journals (hauptsächlich US-amerikanischer und britischer Provenienz) führt zu wenig wünschenswerten Streamlining-Effekten. So kann die Reproduktion ähnlicher Auffassungen und Tendenzen die Konformität von Wissen befördern. Dadurch besteht die Gefahr einer strukturellen Diskriminierung oder Ausgrenzung von Ansätzen, die durch kreative und innovative Offenheit zur Erarbeitung (neuen Wissens) beitragen. Das bedeutet für viele geistes-, kultur- und sozialwissenschaftliche Fächer eine massive strukturelle Benachteiligung, die auch den Diversitätsbemühungen entgegenläuft, wenn die Publikationskulturen unterschiedlicher Fachtraditionen zusehends marginalisiert und egalisiert werden. Eine auf diese Weise formalisierte Einschränkung der Bewertung wissenschaftlicher Leistungen unter Massgabe einer vermeintlich systemischen Vergleichbarkeit ist inakzeptabel.

Die Diskussion um wissenschaftliche Publikationsplattformen und Praktiken vor dem Hintergrund etablierter Qualitätssicherungssysteme ist fachlich und sachlich notwendig. Der Ausbau von Fachzeitschriften zur weiteren akademischen und erkenntnisbezogenen Vernetzung ist ausdrücklich zu begrüssen. Die Gesellschaft für Medienwissenschaft und die unterstützenden Fachgesellschaften wollen zu einer kritischen Debatte über die zunehmende strukturelle Eingrenzung, Engführung und Normierung eines qualitätsgesicherten Publizierens aufrufen. Das breite Spektrum von Qualitätsstandards wissenschaftlicher Publikationen und deren Formen sind im Sinne einer differenzierten Wissenschaftskultur uneingeschränkt anzuerkennen.

Grundsätzlich sollte die Bewertung wissenschaftlicher Leistungen (etwa bei Drittmittelanträgen, Berufungen oder Evaluationen) auf der Basis der Inhalte selbst und nicht entlang intransparenter und wenig vergleichbarer (Publikations-)Kennzahlen erfolgen. 
Wir appellieren an alle Beteiligten im nationalen und internationalen Wissenschaftsbetrieb sowie in der Hochschul- und Forschungsförderpolitik, die Offenheit fachlich diverser Veröffentlichungstraditionen und auch nicht-englischsprachige Publikationskulturen anzuerkennen und nachhaltig gleichwertig zu berücksichtigen.

In diesem Zusammenhang wollen wir eine offene Diskussion der Bedeutung von diversen Publikationskulturen im Wissenschaftsbetrieb anregen und fordern die Einrichtung interdisziplinärer Reformkommissionen unter Einbindung von Vertretenden geistes-, kultur- und sozialwissenschaftlicher Fachgesellschaften und der Hochschulleitungen sowie der zuständigen Ministerien und des Wissenschaftsrats.

\section{Die Unterzeichnenden:}

- Gesellschaft für Medienwissenschaft (GfM).

- Sektion Medienpädagogik in der Deutschen Gesellschaft für Erziehungswissenschaft (DGfE).

- Sektion Medienpädagogik der Österreichischen Gesellschaft für Forschung und Entwicklung im Bildungswesen (ÖFEB).

- Gesellschaft für Theaterwissenschaft. 